\title{
Evidence of an increased pathogenic footprint in the lingual microbiome of untreated HIV infected patients
}

Angeline T Dang ${ }^{1,4}$, Sean Cotton ${ }^{2}$, Sumathi Sankaran-Walters ${ }^{1}$, Chin-Shang Li ${ }^{3}$, Chia-Yuan Michael Lee ${ }^{5}$, Satya Dandekar ${ }^{1}$, Bruce J Paster ${ }^{2,6}$ and Michael D George ${ }^{1 *}$

\begin{abstract}
Background: Opportunistic oral infections can be found in over $80 \%$ of HIV + patients, often causing debilitating lesions that also contribute to deterioration in nutritional health. Although appreciation for the role that the microbiota is likely to play in the initiation and/or enhancement of oral infections has grown considerably in recent years, little is known about the impact of HIV infection on host-microbe interactions within the oral cavity. In the current study, we characterize modulations in the bacterial composition of the lingual microbiome in patients with treated and untreated HIV infection. Bacterial species profiles were elucidated by microarray assay and compared between untreated HIV infected patients, HIV infected patients receiving antiretroviral therapy, and healthy HIV negative controls. The relationship between clinical parameters (viral burden and CD4+ T cell depletion) and the loss or gain of bacterial species was evaluated in each HIV patient group.

Results: In untreated HIV infection, elevated viremia was associated with significantly higher proportions of potentially pathogenic Veillonella, Prevotella, Megasphaera, and Campylobacter species in the lingual microbiome than observed in healthy controls. The upsurge in the prevalence of potential pathogens was juxtaposed by diminished representation of commensal Streptococcus and Veillonella species. Colonization of Neisseria flavescens was lower in the lingual microbiome of HIV infected patients receiving antiretroviral therapy than in uninfected controls.

Conclusions: Our findings provide novel insights into the potential impact of HIV infection and antiretroviral therapy on the community structure of the oral microbiome, and implicate potential mechanisms that may increase the capacity of non-commensal species to gain a stronger foothold.
\end{abstract}

Keywords: HIV, Microbiota, Oral mucosa, Dysbiosis, CD4+ T cells, Streptococcus, Commensal, Microbiome, HOMIM

\section{Background}

Human immunodeficiency virus (HIV) infection leads to a progressive loss of CD4+ T cell numbers and function, impairing immune responses and rendering the host susceptible to secondary opportunistic infections [1-3]. Opportunistic infections (OI) of the oral mucosa are presented in up to $80 \%$ of HIV-infected patients [4], often causing debilitating lesions that contribute to deterioration in nutritional health. While, several studies

\footnotetext{
* Correspondence: mdgeorge@ucdavis.edu

'Dept. of Medical Microbiology and Immunology, University of California, Davis, Davis, CA, USA

Full list of author information is available at the end of the article
}

have examined the effects of HIV infection on oral mucosal immunity in patients with OI [5,6], questions regarding the role of epithelial pathogenesis remain to be answered. Although the underlying mechanisms remain unknown, the oral epithelium appears to be more permeable and perturbed during HIV infection [7]. Studies in the simian immunodeficiency virus (SIV) nonhuman primate model may provide some mechanistic clues. Similar to the intestinal mucosa [8,9], SIV infection leads to a rapid down regulation of genes that mediate oral epithelial regeneration [10]. In addition to increasing barrier permeability, impairment of epithelial regenerative capacity is likely to enhance susceptibility

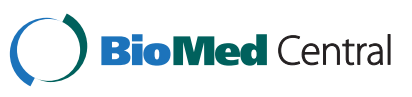


to OI by disrupting homeostatic interactions with the overlying protective microbiota (microbiome).

The human oral microbiome is a complex polymicrobial community in delicate balance. The microbiota consists of a variety of aerobic and anaerobic species that produce a milieu of peptides and polysaccharides that interact with each other and with host molecules to maintain a stable symbiotic microenvironment [11-13]. More than 700 bacterial species have been detected in the human oral cavity, of which $35 \%$ are, so far, uncultivable [14]. In healthy oral tissues, access to the epithelium is vigorously protected from non-commensal organisms, due in part to the physical and physiological barriers supplied by the microbiome [15]. Microbial antigens such as lipopolysaccharide, flagellin, peptidoglycan, and fimbrae presumably contribute to this process as well. These antigens differentially stimulate innate response mechanisms through pattern recognition receptors (PRRs) and thereby regulate the local physiological environment. In turn, the physiological constraints dictate the corresponding profile of organisms the epithelial surface can support $[16,17]$. Although appreciation for the putative role that the microbiome can play in the initiation and/or enhancement of oral disease has grown considerably in recent years, little is known about the impact of HIV infection on host-microbe interactions within the oral cavity.

In the present study we provide, to our knowledge, the first characterization of modulations in the dorsal tongue (lingual) microbiota that are associated with chronic HIV infection. Lingual bacterial species were identified in oral swab samples utilizing the Human Oral Microbe Identification Microarray, or HOMIM (http:// mim.forsyth.org/). Bacterial species profiles were compared between untreated chronically HIV infected patients, chronically HIV infected patients receiving antiretroviral therapy (ART), and healthy uninfected age matched controls. CD4+ T cell depletion and viral burden were measured in peripheral blood by flow cytometry and Amplicor viral load assays, respectively. Our findings provide novel insights into the impact of HIV infection on host-microbe homeostasis within the lingual microbiome, and reveal a potential correlation between high viremia and colonization of several putative opportunistic pathogens in untreated patients.

\section{Results}

HIV infected patients and healthy controls harbor similar quantities of lingual bacteria

To characterize alterations in the oral microbiome associated with chronic HIV infection and administration of antiretroviral therapy (ART), resident bacterial species profiles on the dorsal tongue epithelium were compared between 12 HIV infected patients (6 ART naïve, 6 receiving ART) and 9 healthy HIV-negative controls. The dorsal tongue surface was chosen for microbiome sampling because that anatomical site typically displays less sample to sample variation in microbial community structure compared to other oral niches, and because it is a common location for manifestation of HIV associated oral disease (e.g. candidiasis). One of the $6 \mathrm{HIV}$ infected subjects on ART (\#166) had a previous case of thrush, diagnosed 2-3 weeks prior to collection of the oral swab sample, but was not symptomatic or undergoing antibiotic treatment at the time of sample collection. None of the other treated or untreated HIV infected patients in the study had been previously or concurrently diagnosed with candidiasis or any other HIV associated oral complications. In addition, none of the HIV infected or control subjects were under concurrent antibiotic or antimycotic treatment during the study.

Patients in the ART naive group had been HIV positive for at least one year, and displayed peripheral viral loads ranging from $9 \times 10^{3}$ to $2 \times 10^{4}$ RNA copies $/ \mathrm{mL}$ blood and CD4+ T cells counts ranging from 525 to 137 cells/ $\mathrm{mL}$ blood (Table 1). All HIV patients in the treated group had been receiving ART uninterrupted for at least 3 years, showed undetectable peripheral viral loads, and had CD4+ T cell counts in that ranged from 322 to 1069 cells/ $\mathrm{mL}$ blood. Peripheral CD4+ T cell depletion was statistically significant in the untreated HIV infected group when compared to uninfected healthy controls (Figure 1A). HIV patients receiving long-term ART showed significantly higher $\mathrm{CD} 4+\mathrm{T}$ cell numbers than untreated patients, although not reaching the levels observed in healthy controls. CD4/CD8 ratios in untreated HIV patients and HIV patients on ART were both significantly below the levels observed in healthy controls (Figure 1B).

To evaluate the general impact of chronic HIV infection and ART administration on the oral microbiota, we utilized the HOMIM to identify the number of bacterial species residing on the dorsal tongue surface of HIV infected patients and compared the species profiles to healthy controls. We first compared the mean number of bacterial species (all probes with signals $\geq 1$ ) detected on the tongue between each patient and control group (Species Score) and found no significant differences (Figure 2A). To determine if there were differences in the total number of bacteria on the tongue (Bacterial Load), the total integer score for each sample was then tallied over all the probes on the array and mean values were compared between controls and HIV infected groups. Similar to the Species Score, no statistically significant difference was detected in Bacterial Load between uninfected and infected groups (Figure 2B). In addition, we found that Species Score and Bacterial Load data were highly correlated in individual samples across all experimental groups and controls (Figure 2C). Although the Species Score and Bacterial 
Table 1 Study Participants

\begin{tabular}{|c|c|c|c|c|c|c|c|c|c|c|}
\hline & \multirow{2}{*}{$\begin{array}{l}\text { Patient } \\
\text { ID }\end{array}$} & \multirow[b]{2}{*}{ Gender } & \multirow[b]{2}{*}{ Status } & \multirow[b]{2}{*}{ ART } & \multirow[b]{2}{*}{$\mathrm{CDA}^{*}$} & \multirow[b]{2}{*}{$\mathrm{CDA}^{*}$} & \multirow[b]{2}{*}{ VL+ } & \multicolumn{3}{|l|}{ Time } \\
\hline & & & & & & & & HIV+ & Time Tx & Ag69e \\
\hline \multirow[t]{9}{*}{ HIV Control $(n=9)$} & 204 & $\mathrm{~F}$ & Control & $\mathrm{N} / \mathrm{A}$ & 730 & 327 & N/A & N/A & $\mathrm{N} / \mathrm{A}$ & 54 \\
\hline & 206 & F & Control & N/A & 510 & 275 & N/A & N/A & N/A & 69 \\
\hline & 213 & $\mathrm{~F}$ & Control & $\mathrm{N} / \mathrm{A}$ & 1021 & 382 & $\mathrm{~N} / \mathrm{A}$ & N/A & $\mathrm{N} / \mathrm{A}$ & 43 \\
\hline & 214 & F & Control & N/A & 1559 & 1294 & N/A & N/A & N/A & 36 \\
\hline & 215 & M & Control & N/A & 380 & 290 & N/A & N/A & N/A & 57 \\
\hline & 218 & M & Control & N/A & 674 & 241 & N/A & N/A & N/A & 35 \\
\hline & 222 & $F$ & Control & N/A & ND & ND & N/A & N/A & N/A & 24 \\
\hline & 225 & $F$ & Control & N/A & ND & ND & N/A & N/A & N/A & 55 \\
\hline & 226 & F & Control & N/A & 1114 & 401 & N/A & N/A & N/A & 61 \\
\hline \multirow[t]{6}{*}{ HIV ART- $(n=6)$} & 217 & M & $\mathrm{HIV+}$ & No & 307 & 1117 & 216000 & $9 \mathrm{yr}$ & None & 39 \\
\hline & 224 & F & $\mathrm{HIV}+$ & No & 238 & 1072 & 90000 & $3 \mathrm{yr}$ & None & 39 \\
\hline & 207 & $\mathrm{~F}$ & $\mathrm{HIV+}$ & No & 151 & 385 & 55000 & $1.5 \mathrm{yr}$ & None & 59 \\
\hline & 221 & M & HIV+ & No & 381 & 1188 & 50000 & $9 \mathrm{yr}$ & None & 41 \\
\hline & 223 & $\mathrm{~F}$ & HIV+ & No & 137 & 389 & 18000 & $1 \mathrm{yr}$ & None & 30 \\
\hline & 212 & M & $\mathrm{HIV+}$ & No & 525 & 873 & 9000 & $1.5 y$ & None & 28 \\
\hline \multirow[t]{6}{*}{$H I V+A R T+(n=6)$} & 158 & M & $\mathrm{HIV+}$ & Yes & 873 & 1302 & UD & $23 \mathrm{yr}$ & $20 \mathrm{yr}$ & 58 \\
\hline & 166 & M & HIV+ & Yes & 540 & 1041 & UD & $13 \mathrm{yr}$ & $13 \mathrm{yr}$ & 53 \\
\hline & 205 & $F$ & $\mathrm{HIV+}$ & Yes & 1069 & 582 & UD & $15 \mathrm{yr}$ & $8 \mathrm{yr}$ & 61 \\
\hline & 208 & $\mathrm{~F}$ & $\mathrm{HIV+}$ & Yes & 322 & 311 & UD & $6 \mathrm{yr}$ & $3 \mathrm{yr}$ & 37 \\
\hline & 219 & $F$ & $\mathrm{HIV}+$ & Yes & 490 & 531 & UD & $6 \mathrm{yr}$ & $6 \mathrm{yr}$ & 44 \\
\hline & 228 & M & $\mathrm{HIV+}$ & Yes & 368 & 582 & UD & $9 \mathrm{yr}$ & $9 \mathrm{yr}$ & 39 \\
\hline
\end{tabular}

Clinical characteristics of study participants. ${ }^{*} \mathrm{CD} 4+, \mathrm{CD} 8+\mathrm{T}$ cells $/ \mathrm{mL}$ blood. ${ }^{+} \mathrm{HIV}$ RNA copies $/ \mathrm{mL}$ blood. ART $=$ antiretroviral therapy, $\mathrm{Tx}=$ treatment.

Load data does not address proportional shifts in bacterial species between experimental groups and controls, the findings do indicate that the capacity of the lingual epithelium to support complex polymicrobial communities was not impaired by chronic HIV infection or the administration of ART.

\section{Modulations in the lingual microbiome of HIV infected patients}

To evaluate whether HIV infection was associated with alterations in the community structure of the lingual microbiota in HIV patients, we next analyzed the phylogenetic distribution of species that were detected in the
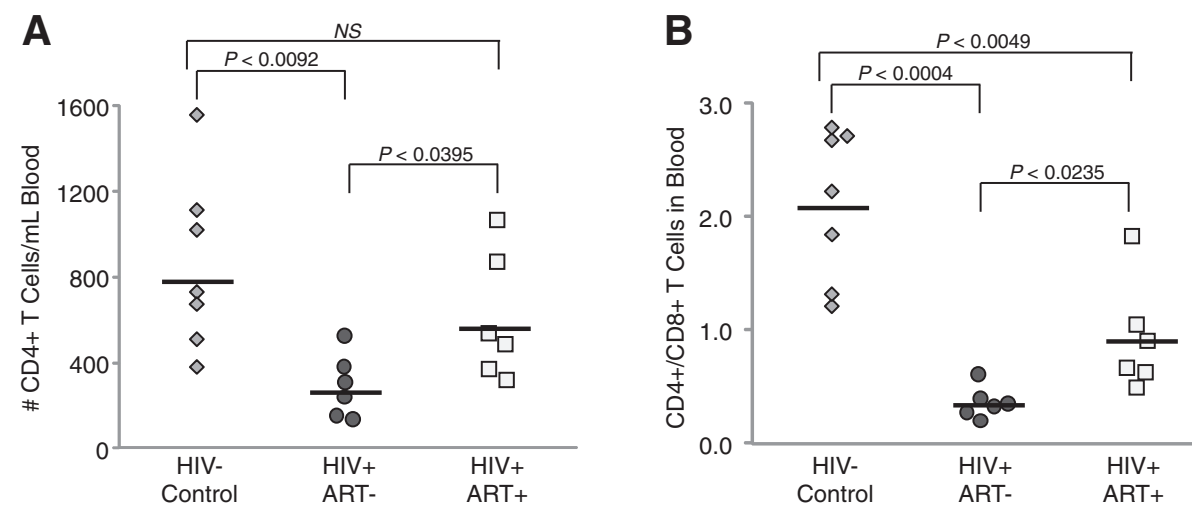

Figure 1 Comparison of circulating CD4+ and CD8+ T cell subsets. The absolute quantity of CD4+ T cells $(\mathbf{A})$, and the ratio of CD4+ and CD8+ T cells (B) as reported in CBC analysis of the blood of HIV- controls, untreated HIV infected patients, and HIV infected patients receiving ART. 

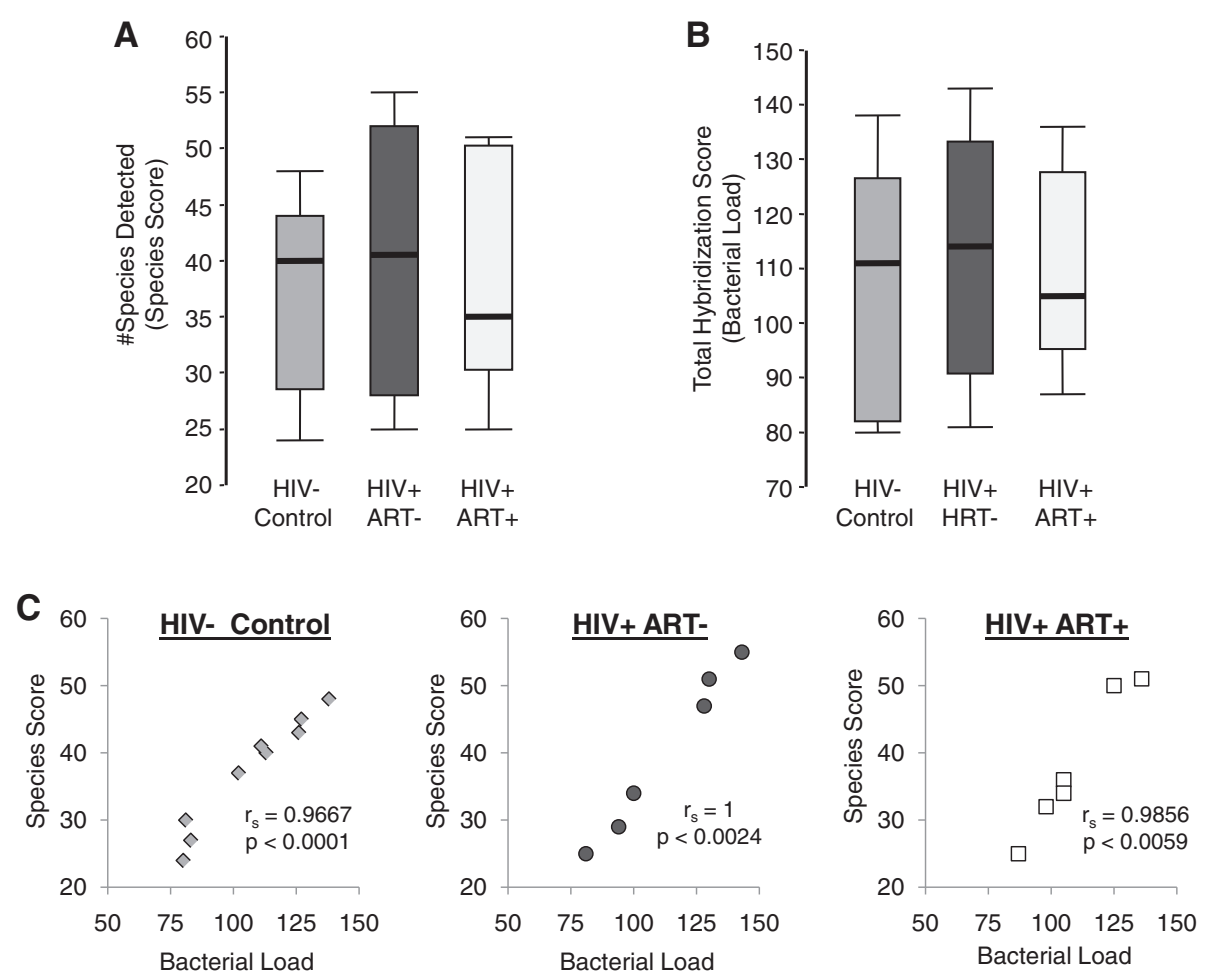

Figure 2 HOMIM-based analysis of bacterial growth in the lingual microbiome. (A) Comparison of the number of bacterial species (Species Score) detected by HOMIM assay on the tongue epithelium of healthy HIV-controls, ART naive chronically HIV infected patients, and HIV infected patients on ART. Median values are shown in horizontal bars. (B). HOMIM-based comparison of total bacterial populations (Bacterial Load) on the tongue epithelium of HIV- controls and HIV + patient groups. (C) Correlation between Species Score and Bacterial Load data as determined by Spearman rank correlation coefficient analysis.

majority of subjects in each patient group (Figure 3). As observed in previous studies, Streptococcus species dominated the oral microbiome of healthy subjects [1821], comprising $\sim 38 \%$ of all species detected by HOMIM, followed by Veillonella ( $19 \%$ of all species) and Rothia ( 7\% of all species). In total, 11 different genera were represented in the oral microbiome of at least one-half of all healthy controls. In contrast, 14 genera were detected in ART naive HIV infected patients, which included all of the genera detected in healthy

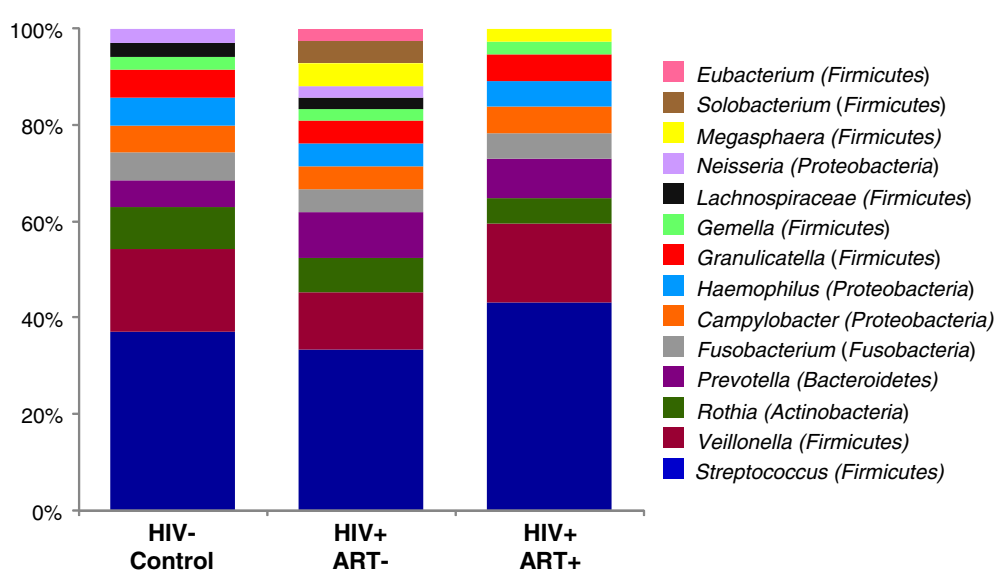

Figure 3 Proportional taxonomic assignments at the genus level in controls and HIV + patient groups. The relative proportions of the genera detected in the total lingual bacterial community in a majority of healthy controls, untreated HIV infected patients, and HIV patients on ART are represented by the height of their individual bars in the stacked bar graphs. Untreated HIV patients displayed an overall increase in genus representation, while HIV patients on ART showed a modest reduction. 
controls as well as Megasphaera, Eubacterium, and Solobacterium. Notably, higher representation of these 3 genera appeared to be counterbalanced by lower relative proportions of core commensal Streptococcus and Veillonella species. Thus, the taxonomic findings also supported our observations that the "Bacterial Load" and "Species Score" generated by HOMIM analysis did not differ significantly between treated or untreated HIV infected groups and healthy controls.

Recent studies suggest that long-term ART may have adverse effects on the oral health of HIV infected patients [22]. In comparison to controls and untreated HIV patients, only 10 genera were represented in the oral microbiome of HIV patients undergoing ART. Representation from Lachnospiraceae and Neisseria was largely lost, while similar to the untreated HIV + group, Megasphaera colonization was higher than observed in healthy subjects. While not reaching statistical significance, the loss in prevalence of Neisseria flavescens was most striking, colonizing the microbiome of $67 \%$ of uninfected controls and untreated HIV patients, but only 17\% (one subject) of HIV patients on ART. These data may be notable in light of reports that have linked reduced oral colonization by $N$. flavescens with increased incident of caries [23]. In agreement with Bacterial Load findings (Figure 2B), the lower relative proportions of Lachnospiraceae and Neisseria observed in the microbiome of HIV patients on ART appeared to be counterbalanced by higher relative proportions of other genera. In addition to Megasphaera, HIV patients on ART showed substantially higher colonization of Streptococcus species when compared to healthy controls and the ART naive HIV + group. Collectively, these findings indicate that administration of ART may lead to alterations in the phylogenetic profile of the oral microbiota that are fundamentally distinct from the changes associated with untreated HIV infection.

\section{Association between HIV burden and colonization by potential opportunistic pathogens}

When the phylogenetic distribution of oral bacteria was evaluated in each patient individually, a substantial amount of variability within the experimental groups and controls was revealed (Figure 4). However, despite this variability, the phylogenetic profiles of 3 of the untreated HIV infected patients $(207,217$, and 224 - labelled in red text) were strikingly similar. Further examination revealed that these 3 patients also displayed the highest levels of viral burden in our study cohort, and that each of the patients had $<350 \mathrm{CD} 4+\mathrm{T}$ cells/ $\mathrm{mL}$ of blood. Correlative analyses were then performed to evaluate the potential relationship between clinical parameters (viral replication and CD4+ T cell depletion) and modulations in the oral microbiome (Bacterial Load and Species Score data). Although data from additional samples will be needed to validate the trend, we observed a potential correlation between peripheral viral

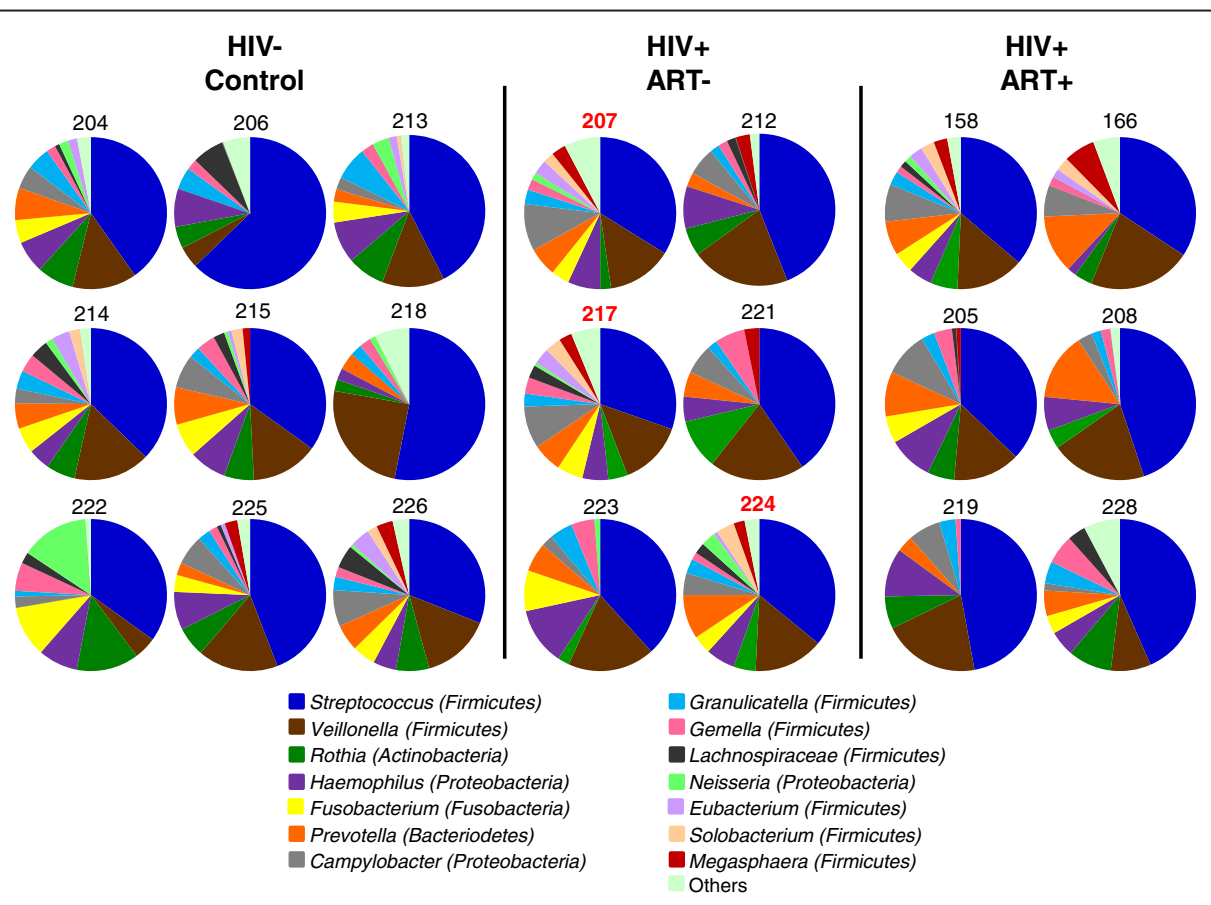

Figure 4 Proportions of taxonomic assignments at the genus level in individual control subjects and HIV + patients. The relative proportions of the genera detected in the total lingual bacterial community of each study participant are represented in pie charts. Similar genus distribution profiles were identified in 3 untreated HIV infected patients (207, 217, and 224: labelled in red text). 
loads and Bacterial Load in ART naive HIV infected patients (Figure 5). A similar potential correlation was also observed between viral loads and Species Score (data not shown). Depletion of CD4+ T cells in the untreated HIV + group showed a similar but weaker trend towards correlation with Bacterial Load and Species Score. However, as with viral loads, high standard deviations associated with relatively small sample sizes prevented us from definitively linking CD4+ T cell depletion with differences in the oral microbiota between untreated HIV patients and healthy controls.

We next analyzed differences in the prevalence of individual bacterial species between untreated HIV infected patients and healthy controls. Although differences in the abundance of several species approached statistical significance when comparing the untreated HIV infected group as a whole to controls, these differences often became significant when comparing HIV infected patients with high viral loads (HVL). We defined HVL, for the purposes of our study, as viral burden $\geq 50 \mathrm{~K}$ HIV copies/mL blood. Veillonella parvula was the lone exception, displaying a significant difference in abundance $(P=0.042)$ from uninfected controls across the entire untreated HIV infected group (Figure 6A). We detected significant differences between HVL HIV patients and uninfected controls in the prevalence of Campylobacter concisus and/or Campylobacter rectus [cross-hybridizing HOMIM probe] $(P=0.032)$, Prevotella pallens $(P=0.027)$, and Megasphaera micronuciformis $(P=0.031)$ (Figures 6B-6D). Interestingly, most of the species displaying higher prevalence in HVL HIV patients have also been linked to periodontal pathogenesis, and M. micronuciformis has been identified in previous studies through its association with serious clinical infections [24]. Taken together, the presence of potential bacterial pathogens at higher abundance and commensal Streptococcus and Veillonella species at

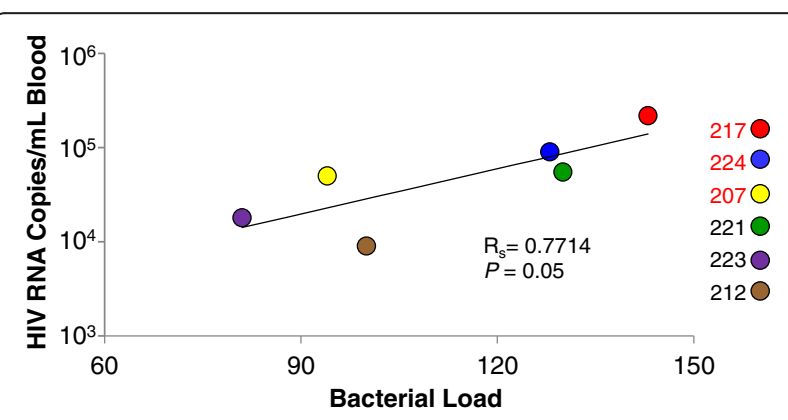

Figure 5 Relationship between HIV burden and increased bacterial growth in the oral microbiome. The relationship between viral loads in peripheral blood and the gain of bacterial growth (Bacterial Load score identified by HOMIM analysis) in ART naïve HIV infected patients was determined by Spearman rank correlation coefficient analysis. HIV infected patients that showed similar oral microbiome profiles are labelled in red text. lower abundance than healthy controls indicates that uncontrolled HIV infection may be associated with a fundamental shift in the character of host-microbe interactions within the oral microbiome.

\section{Discussion}

Maintenance of oral health is dependent on preserving the homeostatic balance between host and the distinct microbial communities that colonize the various anatomical microenvironments in the oral cavity. HIV infected patients often display increased susceptibility to opportunistic oral infections that are presumably linked, in part, to disruption of host-microbe homeostasis (dysbiosis). In the current study, we utilize HOMIM-based analyses to characterize and compare the bacterial composition of the lingual microbiome in a relatively small, but well-defined cohort of untreated chronically HIV infected patients $(n=6)$, HIV patients on ART $(n=6)$, and uninfected controls $(n=9)$. Due to the small sample sizes, it is important to caution that our findings represent a preliminary indication of the impact of HIV infection on the community structure of the oral microbiome. Indeed, the microbiome of even a single individual can be difficult to define, consisting of entrenched endogenous species and transient species whose prevalence can vary depending on time of sampling, diet, oral hygiene, and numerous other parameters [19]. Extensive cross sectional and longitudinal sampling of patients with and without oral manifestations will ultimately be necessary to fully characterize the role of the microbiota in HIV associated oral pathogenesis. The current study represents an important first step towards that goal. Our findings indicate that chronic HIV infection may lead to substantial disruptions in the community structure of the lingual microbiota, even in the absence of clinical oral manifestations.

Several potential mechanisms that have been revealed in previous studies may contribute to the development of host-microbe dysbiosis in the oral mucosa during immunodeficiency virus infection. Recently, analysis of SIV infected rhesus macaques demonstrated that, similar to the gut mucosa, depletion of CD4+ T cells from the oral mucosa is rapid and dramatic [10]. This finding underscores the likelihood that immune dysfunction resulting from the loss of CD4+ T cell activity in the oral cavity could contribute to the development of oral manifestations during SIV/HIV infection. Recent studies suggest that Notch-1 signaling mediates epithelial barrier function in the gut through interaction with $\mathrm{CD} 4+\mathrm{T}$ cells [25]. Although interaction of Notch-1 with CD4+ T cells has not been studied in the oral mucosa, Notch-1 signaling is known to mediate oral epithelial cell differentiation [26]. Thus, it is possible that $\mathrm{CD} 4+\mathrm{T}$ cell depletion from the oral mucosa of HIV infected subjects 

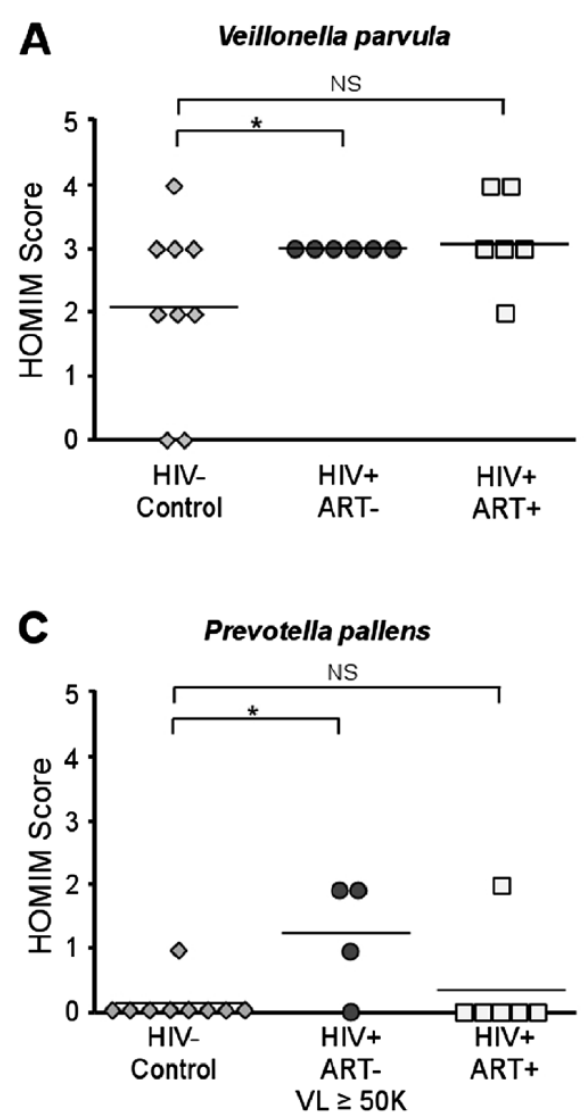
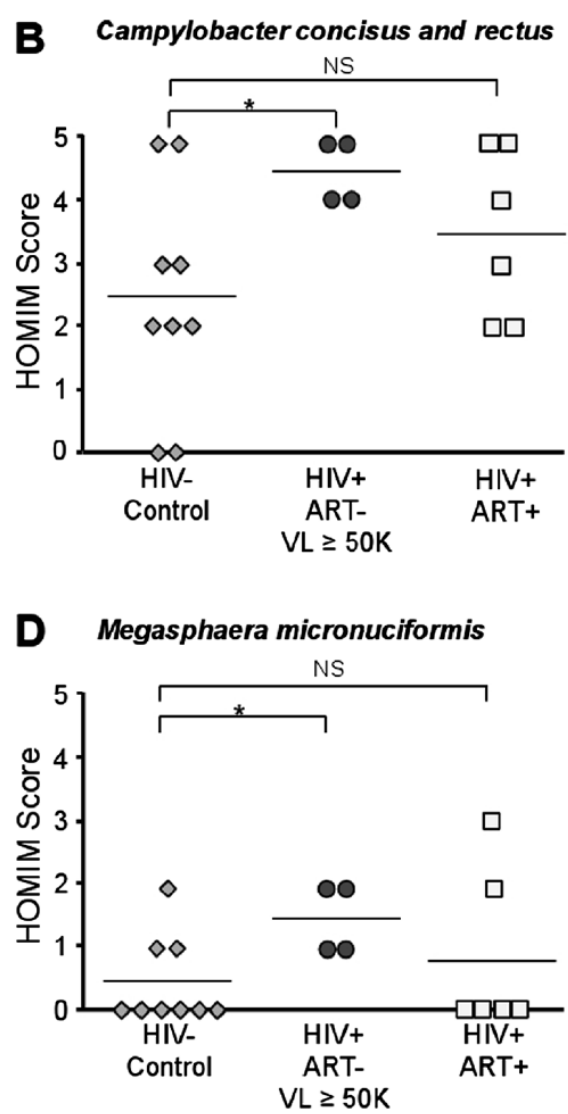

Figure 6 Emergence of opportunistic pathogens in the oral microbiome of ART naive HIV infected patients. (A) A statistically significant increase in the growth of Veillonella parvula was detected amongst all untreated HIV + subjects, while growth of (B) Campylobacter concisus/rectus, (C) Prevotella pallens, and (D) Megasphaera micronuciformis was significantly increased in untreated patients with HIV loads $\geq 50 \mathrm{~K} / \mathrm{mL}$ of blood. Statistical analysis was performed using Wilcoxon rank-sum tests.

may also lead to the impairment of epithelial growth and, by extension, host-microbe dysbiosis.

In addition, depletion of the Th17 subset of CD4+ T cells has been shown in the gut mucosa impair response to microbial infections [8,27], in part by dampening expression of epithelial antimicrobial peptides [28]. HIV patients display decreased expression of histatin-5, a potent antimycotic known to inhibit the growth of Candida albicans [29]. Moreover, in vitro studies suggest that X4-tropic HIV can inhibit expression of human beta defensin-2 (hBD-2) and other innate immune factors in differentiated oral epithelium [30]. Because hBD-2 functions as a chemoattractant for dendritic cells in addition to its antimicrobial activity [31], the loss of hBD-2 during HIV infection could potentiate the colonization of pathogenic species through multiple mechanisms. Thus, it is conceivable that, similar to the gut mucosa, Th17 cells may be depleted from the oral mucosa in SIV/HIV infection, thereby providing a potential mechanism for increased susceptibility to dysbiosis and infection from C. albicans and other non-commensal pathogens.
Interestingly, one of the largest and most consistent alterations we detected in the oral microbiome of untreated HIV patients was a shift in the representation of Veillonella species. Although the relative percentage of Veillonella dropped from $\sim 19 \%$ of the total lingual bacterial population in healthy controls to just over $10 \%$ in untreated HIV infected subjects, that same group displayed a uniform increase in the growth of $V$. parvula. While $V$. parvula is a commensal gram negative anaerobic coccus in healthy individuals [32], it is also the only known Veillonella species associated with oral disease. V. parvula has been implicated in severe early childhood caries [33], primary endodontic infections [34], and other periodontal diseases [35]. Recent studies indicate that V. parvula lipopolysaccharide (LPS) stimulates pro-inflammatory cytokine production and $\mathrm{p} 38$ MAPK activation through TLR-4 dependent mechanisms [36]. Thus, it is possible that increased $V$. parvula colonization (as well as other opportunistic pathogens) could establish an inflammatory environment in the oral cavity, that in turn, contributes to the chronic inflammation and immune activation that 
characterizes HIV disease progression. Future studies are warranted to determine whether increased colonization of putative periodontal pathogens on the tongue epithelium reflects similar increased growth in gingival and subgingival tissues, and perhaps a systemic distribution to more distal mucosal compartments.

\section{Conclusions}

In summary, we identify statistically significant increases in the growth of $V$. parvula, P. pallens, C. rectus and/or C. concisus, and M. micronuciformis in the lingual microbiome of untreated HIV infected subjects, albeit a small patient cohort where previous oral infections could not be considered. Due to advances in therapeutic efficacy and clinical care in developed countries, susceptibility of HIV patients to opportunistic oral infections has been dramatically reduced $[37,38]$. However, worldwide, where the vast majority of HIV infected individuals do not have access to basic clinical care or therapy, oral complications remain a serious problem $[39,40]$. Largescale sampling from an appropriate range of geographic and cultural regions and collation of data from multiple studies will lead to a more complete understanding of host-microbe dysbiosis in HIV infection. To that end, the HOMIM and similar high throughput methodologies designed for rapid identification of microbial profiles may represent ideal cost-effective tools for accomplishing such ambitious large-scale endeavors.

\section{Methods}

\section{Patients and sample collection}

All participants were enrolled through the Center for AIDS Research, Education and Services (CARES) clinic in Sacramento, CA after providing informed written consent. The research was carried out according to Institutional Review Board (IRB)-approved procedures (219139-5) and in compliance with the Helsinki Declaration. The oral health status of each patient was determined prior to participation in the study, including any recent or concurrent periodontal procedures and history of candidiasis and other oral infections. Patients undergoing antibiotic or antimycotic treatment were excluded from the study. Pertinent clinical data was also obtained on all participants. These data included duration of HIV infection, CBC with differential, CD4+/CD8+ T cell numbers (blood was not collected from 2 of the 9 uninfected control subjects), peripheral blood HIV viral loads, and duration of antiretroviral therapy. Peripheral blood viral load assays were performed at the CARES clinical lab using the Amplicor HIV-1 Assay (Roche Molecular Diagnostics). Two-sided Satterthwaite's and Student's t-tests were utilized to determine the statistical significance of differences in $T$ cell subsets between uninfected controls and HIV infected patient groups.
During the same clinical appointment that blood samples were obtained, tongue epithelial samples were collected utilizing non-invasive swabbing of the dorsal surface. Briefly, MasterAmp Buccal Swabs $\odot$ (Epicentre Biotechnologies, Inc) were used to collect epithelial cells and resident microbes, and DNA was extracted utilizing the protocols and reagents provided in the Epicentre MasterAmp $\odot$ kit. Extracted DNA was transferred into new tubes and stored at $-20^{\circ} \mathrm{C}$ until HOMIM analysis.

\section{HOMIM processing}

Identification of oral bacterial species and quantitation of their relative proportions was carried out using the Human Oral Microbe Identification Microarray, or HOMIM [41]. Briefly, $16 \mathrm{~S}$ rRNA genes (rDNAs) were PCR amplified utilizing a universal primer set (forward primer, 5'GAG AGT TTG ATY MTG GCT CAG; reverse primer, 5'- GAA GGA GGT GWT CCA RCC GCA), and Cy3dCTP-labeled. Purified, labelled $16 \mathrm{~S}$ amplicons were then hybridized to the printed HOMIM slides at $55^{\circ} \mathrm{C}$ for $16 \mathrm{~h}$. Hybridized slides were washed and dried and Cy3 fluorescence was detected using the GenePix 4000B microarray scanner (Axon) with photomultiplier settings (PMT) of 650 and wavelength of $532 \mathrm{~nm}$.

\section{Analysis of HOMIM data}

Analysis of HOMIM data was performed as previously described [42,43]. Briefly, hybridization spot intensities were converted to one of the 6 integer signal levels ranging from 0 to 5 , with 0 representing undetectable (above background) and 5 being the maximal intensity among all the profiles being compared. The number of bacterial species (Species Score) present in each sample was determined by summation of all probes with detectable signal (integer score $\geq 1$ ), and a qualitative representation of the total bacteria (Bacterial Load) in each sample was estimated by summation of all integer scores. Correlations between "Species Score" and "Bacterial Load" were analyzed using Spearman rank correlation coefficient. Correlations between clinical parameters (viral loads, CD4+ T cell counts) and a gain or loss of oral bacteria were identified by Spearman rank correlation coefficient analysis. Wilcoxon rank-sum tests were utilized to determine if increases or decreases in individual bacterial species in HIV patient groups were statistically significant compared to healthy HIV- controls. The HOMIM data utilized in the study has been deposited in the Gene Expression Omnibus microarray database (Accession\#: GSE38908).

\section{Competing interests}

The author(s) declare that they have no competing interests.

\section{Authors' contributions}

ATD collected samples, extracted DNA for HOMIM analysis, and drafted the manuscript. SC performed HOMIM assays. SS recruited patients for the study and collected samples. CL participated in the design of the study and 
performed statistical analyses. CML performed statistical analyses. SD participated in the design of the study and edited the manuscript. BJP participated in the design and coordination of the study and edited the manuscript. MDG conceived of the study and its design, directed its coordination, and helped to draft the manuscript. All authors read and approved the final manuscript.

\section{Acknowledgements}

The authors would like to thank the clinicians and staff at the Center for AIDS Research and Education (CARES) Clinic in Sacramento, CA for their help in scheduling patient appointments and collecting samples. This study was funded through a pilot grant from the California Research Center for the Biology of HIV in Minorities (CRCBHM). Statistical support was made possible through funding (UL1 RR024146) from the National Center for Research Resources (NCRR)

\section{Author details}

'Dept. of Medical Microbiology and Immunology, University of California, Davis, Davis, CA, USA. ²Dept. of Molecular Genetics, Forsyth Institute, Cambridge, MA, USA. ${ }^{3}$ Dept. of Public Health Services, University of California, Davis, Davis, CA, USA. ${ }^{4}$ Dept. of Microbiology, Immunology, and Molecular Genetics, University of California, Los Angeles, Los Angeles, CA, USA. ${ }^{5}$ Neuroscience Undergraduate Interdepartmental Program, University of California, Los Angeles, Los Angeles, CA, USA. 'Dept. of Oral Medicine, Infection and Immunity, Harvard School of Dental Medicine, Boston, MA, USA

Received: 26 September 2011 Accepted: 12 July 2012

Published: 28 July 2012

\section{References}

1. McCune JM: The dynamics of CD4+ T-cell depletion in HIV disease. Nature 2001, 410(6831):974-979.

2. Egusa H, Soysa NS, Ellepola AN, Yatani H, Samaranayake LP: Oral candidosis in HIV-infected patients. Curr HIV Res 2008, 6(6):485-499.

3. Hazenberg MD, Hamann D, Schuitemaker H, Miedema F: T cell depletion in HIV-1 infection: how CD4+ T cells go out of stock. Nat Immunol 2000, 1(4):285-289.

4. Reznik DA: Oral manifestations of HIV disease. Top HIV Med 2005, 13(5):143-148

5. Myers TA, Leigh JE, Arribas AR, Hager S, Clark R, Lilly E, Fidel PL Jr: Immunohistochemical evaluation of T cells in oral lesions from human immunodeficiency virus-positive persons with oropharyngeal candidiasis. Infect Immun 2003, 71(2):956-963.

6. Leigh JE, McNulty KM, Fidel PL Jr: Characterization of the immune status of CD8+ T cells in oral lesions of human immunodeficiency virusinfected persons with oropharyngeal Candidiasis. Clin Vaccine Immunol 2006, 13(6):678-683.

7. Challacombe SJ, Naglik JR: The effects of HIV infection on oral mucosal immunity. Adv Dent Res 2006, 19(1):29-35

8. Dandekar S, George MD, Baumler AJ: Th17 cells, HIV and the gut mucosal barrier. Curr Opin HIV AIDS 2010, 5(2):173-178.

9. Sankaran S, George MD, Reay E, Guadalupe M, Flamm J, Prindiville T, Dandekar S: Rapid onset of intestinal epithelial barrier dysfunction in primary human immunodeficiency virus infection is driven by an imbalance between immune response and mucosal repair and regeneration. J Virol 2008, 82(1):538-545.

10. George MD, Verhoeven D, Sankaran S, Glavan T, Reay E, Dandekar S: Heightened cytotoxic responses and impaired biogenesis contribute to early pathogenesis in the oral mucosa of simian immunodeficiency virus-infected rhesus macaques. Clin Vaccine Immunol 2009, 16(2):277-281.

11. Donlan RM, Costerton JW: Biofilms: Survival mechanisms of clinically relevant microorganisms. Clin Microbiol Rev 2002, 15(2):167.

12. Foster JS, Palmer RJ, Kolenbrander PE: Human oral cavity as a model for the study of genome-genome interactions. In: 2003. Marine Biological Laboratory 2003, 204(2):200-204.

13. Lewis K: Riddle of biofilm resistance. Antimicrob Agents Chemother 2001 , 45(4):999-1007.

14. Aas JA, Paster BJ, Stokes LN, Olsen I, Dewhirst FE: Defining the normal bacterial flora of the oral cavity. J Clin Microbiol 2005, 43(11):5721-5732.
15. Tlaskalova-Hogenova H, Stepankova R, Hudcovic T, Tuckova L, Cukrowska B, Lodinova-Zadnikova R, Kozakova H, Rossmann P, Bartova J, Sokol D, et al: Commensal bacteria (normal microflora), mucosal immunity and chronic inflammatory and autoimmune diseases. Immunol Lett 2004 93(2-3):97-108.

16. Alexopoulou L, Kontoyiannis D: Contribution of microbial-associated molecules in innate mucosal responses. Cell Mol Life Sci 2005, 62 (12):1349-1358.

17. Kelly D, Conway S: Bacterial modulation of mucosal innate immunity. Mol Immunol 2005, 42(8):895-901.

18. Belda-Ferre P, Alcaraz LD, Cabrera-Rubio R, Romero H, Simon-Soro A, Pignatelli M, Mira A: The oral metagenome in health and disease. ISME J 2012, 6(1):46-56

19. Dewhirst FE, Chen T, Izard J, Paster BJ, Tanner AC, Yu WH, Lakshmanan A, Wade WG: The human oral microbiome. J Bacteriol 2010, 192(19):5002-5017.

20. Mitchell J: Streptococcus mitis: walking the line between commensalism and pathogenesis. Mol Oral Microbiol 2011, 26(2):89-98.

21. Zaura E, Keijser BJ, Huse SM, Crielaard W: Defining the healthy "core microbiome" of oral microbial communities. BMC Microbio/ 2009, 9:259.

22. Nittayananta W, Talungchit S, Jaruratanasirikul S, Silpapojakul K, Chayakul $P$, Nilmanat A, Pruphetkaew N: Effects of long-term use of HAART on oral health status of HIV-infected subjects. J Oral Pathol Med 2010, 39(5):397-406

23. Crielaard W, Zaura E, Schuller AA, Huse SM, Montijn RC, Keijser BJ: Exploring the oral microbiota of children at various developmental stages of their dentition in the relation to their oral health. BMC Med Genomics 2011, 4:22

24. Marchandin H, Jumas-Bilak E, Gay B, Teyssier C, Jean-Pierre H: de Buochberg MS, Carriere C. Carlier JP: Phylogenetic analysis of some Sporomusa sub-branch members isolated from human clinical specimens: description of Megasphaera micronuciformis sp. nov. Int J Syst Evol Microbiol 2003, 53(Pt 2):547-553.

25. Dahan S, Rabinowitz KM, Martin AP, Berin MC, Unkeless JC, Mayer L: Notch1 signaling regulates intestinal epithelial barrier function, through interaction with CD4+ T cells, in mice and humans. Gastroenterology 2011, 140(2):550-559.

26. Casey LM, Lan Y, Cho ES, Maltby KM, Gridley T, Jiang R: Jag2-Notch1 signaling regulates oral epithelial differentiation and palate development. Developmental dynamics: an official publication of the American Association of Anatomists 2006, 235(7):1830-1844.

27. Milner JD, Sandler NG, Douek DC: Th17 cells, Job's syndrome and HIV: opportunities for bacterial and fungal infections. Curr Opin HIV AIDS 2010, 5(2):179-183.

28. Stange J, Hepworth MR, Rausch S, Zajic L, Kuhl AA, Uyttenhove C, Renauld JC, Hartmann S, Lucius R: IL-22 mediates host defense against an intestinal intracellular parasite in the absence of IFN-gamma at the cost of Th17-driven immunopathology. J Immunol 2012, 188(5):2410-2418.

29. Torres SR, Garzino-Demo A, Meiller TF, Meeks V, Jabra-Rizk MA: Salivary histatin-5 and oral fungal colonisation in HIV + individuals. Mycoses 2009, 52(1):11-15

30. Nittayananta W, Hladik F, Klausner M, Harb S, Dale BA, Coombs RW: HIV type 1 fails to trigger innate immune factor synthesis in differentiated oral epithelium. AIDS Res Hum Retroviruses 2009, 25(10):1013-1021.

31. Chertov O, Yang D, Howard OM, Oppenheim JJ: Leukocyte granule proteins mobilize innate host defenses and adaptive immune responses. Immunol Rev 2000, 177:68-78.

32. Rogosa M: The Genus Veillonella. I.General Cultural, Ecological, and Biochemical Considerations. J Bacteriol 1964, 87:162-170.

33. Tanner AC, Mathney JM, Kent RL, Chalmers NI, Hughes CV, Loo CY, Pradhan $\mathrm{N}$, Kanasi E, Hwang J, Dahlan MA, et al: Cultivable anaerobic microbiota of severe early childhood caries. J Clin Microbio/ 2011, 49(4):1464-1474.

34. Sassone L, Fidel R, Fiqueiredo L, Fidel S, Faveri M, Feres M: Evaluation of the microbiota of primary endodontic infections using checkerboard DNA-DNA hybridization. Oral Microbiol Immunol 2007, 22(6):390-397.

35. Hughes CV, Kolenbrander PE, Andersen RN, Moore LV: Coaggregation properties of human oral Veillonella spp.: relationship to colonization site and oral ecology. Appl Environ Microbiol 1988, 54(8):1957-1963.

36. Matera G, Muto V, Vinci M, Zicca E, Abdollahi-Roodsaz S, van de Veerdonk FL, Kullberg BJ, Liberto MC, van der Meer JW, Foca A, et al: Receptor recognition of and immune intracellular pathways for Veillonella parvula lipopolysaccharide. Clin Vaccine Immunol 2009, 16(12):1804-1809. 
37. Nokta M: Oral manifestations associated with HIV infection. Curr HIV/AIDS Rep 2008, 5(1):5-12.

38. Parveen Z, Acheampong E, Pomerantz RJ, Jacobson JM, Wigdahl B, Mukhtar M: Effects of highly active antiretroviral therapy on HIV-1-associated oral complications. Curr HIV Res 2007, 5(3):281-292.

39. Arotiba JT, Arowojolu MO, Fasola AO, Denloye OO, Obiechina AE: Oral manifestation of HIV/AIDS. Afr J Med Med Sci 2006, 35(Suppl):13-18.

40. Feller L, Khammissa RA, Gugushe TS, Chikte UM, Wood NH, Meyerov R, Lemmer J: HIV-associated Kaposi sarcoma in African children. SADJ 2010, 65(1):20-22.

41. Paster BJ, Dewhirst FE: Molecular microbial diagnosis. Periodontol 2009, 51:38-44.

42. Colombo AP, Boches SK, Cotton SL, Goodson JM, Kent R, Haffajee AD, Socransky SS, Hasturk H, Van Dyke TE, Dewhirst F, et al: Comparisons of subgingival microbial profiles of refractory periodontitis, severe periodontitis, and periodontal health using the human oral microbe identification microarray. J Periodonto/ 2009, 80(9):1421-1432.

43. Paster BJ, Russell MK, Alpagot T, Lee AM, Boches SK, Galvin IL, Dewhirst FE: Bacterial diversity in necrotizing ulcerative periodontitis in HIV-positive subjects. Ann Periodontol 2002, 7(1):8-16.

doi:10.1186/1471-2180-12-153

Cite this article as: Dang et al.: Evidence of an increased pathogenic footprint in the lingual microbiome of untreated HIV infected patients. BMC Microbiology 2012 12:153.

\section{Submit your next manuscript to BioMed Central and take full advantage of:}

- Convenient online submission

- Thorough peer review

- No space constraints or color figure charges

- Immediate publication on acceptance

- Inclusion in PubMed, CAS, Scopus and Google Scholar

- Research which is freely available for redistribution 